\title{
Mandatory dispute resolution and the 2006 family law reforms: Use, outcomes, links to other pathways, and the impact of family violence
}

\section{LAWRIE MOLONEY, PhD}

Senior Research Fellow, Australian Institute of Family Studies, Melbourne; Adjunct Professor, School of Public Health, La Trobe University, Melbourne, Victoria

\section{LIXIA QU, MURP}

Research Fellow, Australian Institute of Family Studies, Melbourne, Victoria

\section{KELLY HAND, BA(HONS)}

Research Fellow, Australian Institute of Family Studies, Melbourne, Victoria

\section{JoHn De MaIO, BCOMm(Hons)}

Senior Research Officer, Australian Institute of Family Studies, Melbourne, Victoria

\section{RaE Kaspiew, PhD}

Senior Research Fellow, Australian Institute of Family Studies, Melbourne, Victoria

\section{RUTH WESTON, BA(HONS), MA}

General Manager Research and Principal Research Fellow, Australian Institute of Family Studies, Melbourne, Victoria

\section{MatThew GraY, PhD}

Deputy Director, Research, Australian Institute of Family Studies, Melbourne, Victoria

\section{Members of the Australian Institute of Family Studies Family LaW Evaluation TEAM}

\section{ABSTRACT}

Community-based mandatory family dispute resolution (more generically known as family mediation) is a central plank of the 2006 changes to the Australian family law system. This paper provides an overview of the data on family dispute resolution from the Australian Institute of Family Studies' evaluation of the 2006 changes. It reports on usage rates of family dispute resolution as well as immediate and medium term outcomes, perceived pathways towards resolution and the impact of family violence on both outcomes and pathways. Though the story is a generally positive one, the data also suggest a need for family relationship sector practitioners and family lawyers to engage proactively in assisting those families who are experiencing significant violence and or significant levels of ongoing serious conflict.

Keywords: family law reforms; family dispute resolution; family mediation; family violence; family conflict 
$\mathrm{I}^{\mathrm{n}}$ n 2006, the Australian Government introduced a series of changes to the family law system. These included changes to the Family Law Act 1975 (Cth) through the Family Law Amendment (Shared Parental Responsibility) Act 2006 (Cth) (SPR Act 2006), and changes to the family relationship services system. Family dispute resolution (FDR), introduced as part of the reforms, became mandatory for most new and ongoing post-separation parenting disputes from July 2008. The initiative was designed to reduce the number of children's cases requiring court-based interventions. It was hoped that early intervention that supported separated parents in resolving their dispute together would also go some way to addressing the child-related problems associated with ongoing entrenched parental conflict (McIntosh, 2003).

Following the 2006 changes, there are now essentially two ways in which an individual can make an application to a family law court with respect to a parenting issue. The first involves filing an application pursuant to one of the exceptions to s 60I, the section that mandates FDR as a default position The second involves obtaining a certificate, which a registered FDR practitioner is empowered to issue if a matter is not amenable to resolution through FDR.

This article reports on some of the data and findings regarding FDR arising out of the evaluation of the 2006 family law reforms conducted by the Australian Institute of Family Studies (AIFS) (Kaspiew, Gray, Weston, Moloney, Hand, $\mathrm{Qu}, \&$ the family law evaluation team, 2009), the "AIFS Evaluation". In particular, the article reports on:

- rate of the take-up of FDR and family relationship services

- changes in applications to the family law courts in children and children and property matters

- the immediate and medium-term outcomes of FDR

- FDR clients' perceptions of the main pathways associated with resolution of the dispute, ongoing negotiations, or stalemate
- links between family violence, outcomes of FDR, and FDR processes

\section{TAKE-UP RATES OF FDR AND OTHER FAMILY RELATIONSHIP SERVICES: CHANGES IN APPLICATION RATES TO FAMILY LAW COURTS}

The AIFS Evaluation reported that during the first 3 years following the 2006 changes to the family law system, the use of family relationship services increased considerably across all but one of the services. Between 2006-07 and 2008-09, use of the existing and expanded FDR services increased from 14,299 to 22,447 clients (57\%), while use of Family Relationship Centres (FRCs), which provide the bulk of FDR services, increased from 13,823 to 60,199 clients (336\%). Between 2004-05 and 2008-09, court applications in children and children and property matters decreased by $22 \%$. This suggests that one of the key aims of the reforms, which was to reduce unnecessary litigation over children, appears at this stage to have been achieved.

\section{UPTAKE AND IMMEDIATE OUTCOMES OF FDR AMONG PARENTS SEPARATED AFTER 2006}

About 10,000 parents, all of whom had separated after the reforms, were surveyed 15 months on average after they had separated. Of these, 31\% of fathers and $26 \%$ of mothers reported that they had "attempted family dispute resolution or mediation". This is referred to in the AIFS Evaluation as the narrow definition of FDR/mediation. To the broader question of "whether counselling, mediation or a dispute resolution service had been contacted", $50 \%$ responded in the affirmative. The figures that follow correspond to those who answered the narrower question. Of this group, $9 \%$ were not sure of the outcome and/or were not sure if they had been issued with a certificate. The remainder can be divided into three groups.

- Group 1: About two fifths (39\%) of the parents who attempted FDR or mediation reported reaching an agreement at the time. 
- Group 2: A little under a third (31\%) of the parents who attempted FDR reported that they did not reach agreement at the time but were not issued with a certificate.

- Group 3: Just over a fifth (21\%) of parents who attempted FDR reported that they did not reach agreement and were issued with a certificate at the time.

\section{MEDIUM TERM RESOLUTION OF PARENTING DISPUTES}

Timing is an important issue in the resolution of disputes over children. Agreements achieved at the time that FDR takes place may assist in stabilising matters for the children and for family members. On the other hand, the situation may be too fluid or too uncertain for a responsible agreement to be made at this time. In addition to gathering data on agreements reached as a direct result of FDR, the AIFS Evaluation also sought information on the parenting arrangements at the time the parents were surveyed, an average of 15 months after they had separated. In terms of a "resolution trajectory", we have labeled this as "medium term".

- Group 1: When asked what the situation was at the time of the survey, about three quarters of the group who had reached agreement at FDR reported that arrangements had been sorted out, about a fifth reported that they were "in the process of sorting out arrangements", and 6\% reported that nothing had been sorted out.

- Group 2: When asked what the situation was at the time of the survey, $65 \%$ of the group who had not reached agreement but had not been issued with a certificate reported that arrangements had been sorted out, $23 \%$ said they were "in the process of sorting out arrangements", and $12 \%$ reported that nothing had been sorted out at this time.

- Group 3: When asked what the situation was at the time of the survey, $36 \%$ reported that they had sorted arrangements out, $47 \%$ were still in the process of out sorting arrangements, and $17 \%$ reported that nothing had been sorted out.

\section{REPORTED PATHWAYS TOWARDS RESOLUTION - THOSE WHO HAD SORTED MATTERS OUT AT THE TIME OF THE SURVEY}

Of the first group of parents who had reached agreement at the time they attended FDR and who had reported that arrangements had been sorted out at the time of the survey, just under a $50 \%$ reported that the main pathway to sorting things out was "counselling, mediation, or FDR". A little over a third reported that the main pathway consisted of discussions between themselves, while $10 \%$ nominated lawyers or courts as their main resolution pathway.

Of the second group - the "no agreement/no certificate parents" - who had sorted matters out at the time of the survey, $61 \%$ reported that the main pathway was via "discussions". Lawyers were seen as the main pathway by $13 \%$ of this group and courts by $7 \%$. Only $6 \%$ of this group attributed the resolution mainly to "counselling, mediation, or FDR".

Of the third group of parents - those who had received a certificate - who had sorted out arrangements at the time of the survey, the majority saw courts $(30 \%)$ or lawyers $(26 \%)$ as the main pathway, while $23 \%$ achieved resolution mainly via discussions. Only $9 \%$ of this group nominated "counselling, mediation, or FDR" as their main pathway towards resolution.

\section{REPORTED PATHWAYS TOWARDS RESOLUTION - THOSE WHO WERE STILL IN THE PROCESS OF SORTING THINGS OUT AT THE TIME OF THE SURVEY}

Of the first group of parents who had reached agreement at the time they attended FDR, but who reported that arrangements were still being sorted at the time of the survey, $34 \%$ said this was happening mainly through discussions, $30 \%$ reported it was happening mainly through court or lawyers, and $27 \%$ said the main pathway was "counselling, mediation, or FDR".

Of the second group - the "no agreement/no certificate parents" - who were still in the process 
of sorting matters out at the time of the survey, $61 \%$ reported that the main pathway was via "discussions". Courts or lawyers were seen as the main pathway by $33 \%$ of this group. Only $6 \%$ attributed their main pathway to "counselling, mediation, or FDR".

Of the third group of parents - the "certificate" group - who were still in the process of sorting things out at the time of the survey, a majority reported using courts or lawyers as their main pathway ( $37 \%$ and $30 \%$, respectively). Just under a fifth were mainly using discussions and only 4\% saw "counselling, mediation, or FDR" as their main pathway.

\section{FAMILY VIOLENCE, OUTCOMES, AND FDR PROCESSES}

It is noteworthy that $85 \%$ of respondents who attempted FDR indicated that there had been physical or emotional abuse in the relationship during the period of or before the separation. By way of comparison, amongst all separated parents, $53 \%$ of fathers and $65 \%$ of mothers, had reported some sort of violence. In addition, among all separated parents, $62 \%$ reported friendly or cooperative post separation relationships. Among those who reported emotional abuse, about half were in the "friendly or cooperative" post-separation category. And even among those who reported physical abuse, more than a third gave this response.

It was found that an absence of reported violence increases the chances of reaching agreement at FDR; almost half of the agreement group reported no violence before or after the separation. But while a history of reported violence reduces the chances of agreement being reached, the evaluation found that almost two fifths of those who reported emotional abuse reached agreement at the time they attended FDR process, and a little over a third who reported physical violence also reached agreement at this time. Only $10 \%$ of those who reported no physical violence were issued with certificates. But even among those who reported physical violence or emotional abuse, only about a quarter were issued with a certificate. Unfortunately, a history of at least some violence is the norm among separated parents. But such a history does not in itself appear to rule out the possibility of successful FDR.

These complex findings have been alluded to in a recent paper (Kaspiew, Gray, Weston, Moloney, Hand, $\mathrm{Qu}, \&$ the family law evaluation team, 2010). They suggest that gauging the suitability and efficacy of FDR requires a highly sophisticated analysis of both how couples perceive any violence and abuse that has occurred between them and of what is meant by agreement. With respect to the former issue, a survey of parents attending family relationship services, conducted as another component of the AIFS Evaluation, found that $26 \%$ of respondents attending FDR "sometimes felt afraid of the person they attended the session about during the sessions at the service". At the same time, it was found that more than $70 \%$ of parents reported that they were treated fairly at FDR and over $50 \%$ said they received the help they needed.

We do not know how many of this sample of clients reached agreements at FDR. At the same time, it is clear from these data and from LSSF data analysed above, that agreements made at the time that FDR takes place account for only one part of the FDR story. For example, when we take a medium term perspective and look at what is happening an average of 15 months after separation, it seems that most of those who went through an FDR process and were not issued with a certificate have sorted out matters in relation to their children. But those issued with a certificate are considerably less likely to have sorted things out at this point in time.

\section{BROADER CONTEXTUAL OBSERVATIONS}

Helping separated parents agree on what is best for their children through the provision of effective dispute resolution services is a central policy objective of the 2006 reforms. At the same time, when we consider the pathways data, FDR 
appears to be seen by many parents as one of the experiences and one of the negotiations that are occurring post-separation. The fact that it is often not seen as a main pathway towards resolution, even among many parents who reached agreement during the process, suggests that it is probably helpful to see FDR as one potentially important intervention in what is frequently a highly fluid situation.

We need to increase our knowledge about the conditions under which FDR is most likely to be helpful. It may be, for example, that FDR is more likely to be effective when it is linked to and in harmony with other negotiations that are taking place regarding future parenting arrangements, child support, division of property, etc. (see for example Fehlberg, Smyth, \& Fraser, 2010 in this issue of JFS) In the meantime, the finding that only $6 \%$ of those who reached agreement reported that nothing was sorted out a year or so later, suggests that an agreement reached during the FDR process probably goes a considerable way towards stabalising the conflict for this group of parents.

In addition, the finding that almost two thirds of FDR recipients who did not reach agreement but did not receive a certificate, had sorted matters out an average of 15 months after separating, may suggest that the process was helpful to these parents. It can be argued of course that this is an optimistic interpretation because most of these clients saw discussions between themselves and not FDR as their main pathway towards resolution. The paradox here is that good FDR, like good counselling, empowers clients to do the work themselves. A question that might clarify this issue in possible future research could be something like: "To what extent do you think that FDR made a positive difference in helping you to sort out your parenting arrangements?"

Finally, the data show that those parents issued with a certificate are the most likely of FDR clients to report violence, the most likely to be seeking assistance mainly from courts or lawyers, and the least likely by a considerable margin to have sorted matters out a year or so after separation. These are the cases in which the children are most likely to be in danger of experiencing the debilitating consequences associated with serious entrenched conflict and/or violence. These are also the cases inclined to take up most resources in the long run, and the cases in which proactive efforts between the family relationship sector and legal sector may prove especially fruitful.

\section{References}

Fehlberg, B., Smyth, B., \& Fraser, K. (2010). Prefiling Family Dispute Resolution for financial disputes: Putting the cart before the horse? Journal of Family Studies 16(3) 197-208.

Folger, J., \& Taylor, A. (1984). Mediation. A comprehensive guide to resolving disputes without litigation. San Francisco: Jossey-Bass Publishers.

Kaspiew, R., Gray, M., Weston, R., Moloney, L., Hand, K., Qu. L., \& the family law evaluation team (2009). Evaluation of the family law reforms. Melbourne: Australian Institute of Family Studies.

Kaspiew, R., Gray, M., Weston, R., Moloney, L., Hand, K., Qu. L., \& the family law evaluation team. (2010). Family Violence. Key findings from the evaluation of the family law reforms. Family Matters, 85, 38-48.

McIntosh, J. (2003). Children living with domestic violence: Research foundations for early intervention. Journal of Family Studies, 9(2),19-234.

Moloney, L., \& McIntosh, J. (2004), Childresponsive practices in Australian family law: Past problems and future directions. Journal of Family Studies, 10(1), 71-86.

N O W A V A I L A B L E
Innovative Approaches to Family Violence
Special Issue of Journal of Family Studies - edited by Margot Schofield and Rae Walker
ISBN 978-1-921348-05-1 Volume 14 Issue 2-3 ii+256 pages October 2008
Addresses pressing public health priorities of interpersonal family violence,
in 16 innovative research articles across several cultures.


Reproduced with permission of the copyright owner. Further reproduction prohibited without permission. 